\title{
Occurrence characteristics of branching structures in equatorial plasma bubbles: a statistical study based on all-sky imagers in China
}

\author{
Kun $W_{u^{1,2}}$, JiYao Xu1,2*, YaJun Zhu ${ }^{1,2}$, and Wei Yuan ${ }^{1,2}$ \\ 'State Key Laboratory of Space Weather, National Space Science Center, Chinese Academy of Sciences, Beijing 100190, China; \\ ${ }^{2}$ School of Astronomy and Space Science, University of Chinese Academy of Sciences, Beijing 100049, China \\ Key Points: \\ - Branching structure is a high occurrence phenomenon during the evolution of EPBs but not all EPBs are observed to develop \\ branching. \\ - Occurrence times of BSEPBs vary with local time, and most of the BSEPBs begin to appear between 21:00 and 22:00 LT. \\ - BSEPBs occurrence is related to solar activity and geomagnetic activity.
}

Citation: Wu, K., Xu, J. Y., Zhu, Y. J., and Yuan, W. (2021). Occurrence characteristics of branching structures in equatorial plasma bubbles: a statistical study based on all-sky imagers in China. Earth Planet. Phys., 5(5), 407-415. http://doi.org/10.26464/epp2021044

\begin{abstract}
Branching structure (BS) is a very important phenomenon in the evolution of equatorial plasma bubbles (EPBs), the mechanism of which is widely studied from observation and from simulation. However, occurrence characteristics of branching structure of equatorial plasma bubbles (BSEPBs) have not been well addressed. In this work, we used seven-years (2012-2018) of observations from two all-sky imagers to study occurrence of BSEPBs in detail. These data reveal a high incidence of BS in EPB cases; in particular, most EPBs occurring on days with geomagnetic disturbances exhibited BS. Periods when all EPBs exhibited BS increased significantly in the 2014 solar maximum. Occurrence times of BSEPBs varied with local time; most of the BSEPBs began to appear between 21:00 and 22:00 LT. During the solar maximum, some BSEPBs were observed after midnight. The data also reveal that BSEPBs are characterized primarily by two branches or three branches. Multi-branching appeared only in the solar maximum. EPB events with different coexisting branching structures increased from 2012 to 2014 and decreased from 2014 to 2018 . These results strongly suggest that BSEPB occurrence is related to solar activity and geomagnetic activity, and thus provide a new perspective for future studies of EPBs as well as enriching our understanding of ionospheric irregularity.
\end{abstract}

Keywords: branching structure of equatorial plasma bubble; equatorial plasma bubble; ionospheric irregularity; all-sky imager

\section{Introduction}

The ionosphere usually becomes unstable after sunset, promoting development of some plasma density depletions in the lowlatitudes, which are called equatorial spread $F$ or equatorial plasma bubbles (EPBs). It is generally accepted that EPBs are caused by Rayleigh-Taylor-like instability (Ott, 1978; Ossakow, 1981; Kelley, 2009; Makela and Otsuka, 2012). As EPBs evolve, some exhibit branching structure (BS). The branching structure of equatorial plasma bubbles (BSEPBs) is a very important phenomenon.

Over the past years, equatorial plasma bubbles exhibiting branching structure have been detected by different instruments (e.g. Woodman and La Hoz, 1976; Tsunoda et al., 1982). Woodman and La Hoz (1976) detected BSEPBs in radar observations. Similarly, Tsunoda et al. (1982) observed BSEPBs in ALTAIR radar data. Mendillo and Baumgardner (1982), and Mendillo and Tyler (1983) ob-

Correspondence to: J. Y. Xu, jyxu@spaceweather.ac.cn

Received 03 JUL 2021; Accepted 21 JUL 2021.

Accepted article online 16 AUG 2021.

C 2021 by Earth and Planetary Physics. served the BSEPB phenomenon repeatedly in airglow observations. Meanwhile, some satellite observations confirmed the presence of branching in EPBs (Aggson et al., 1996). Based on so many observations, generation of BSEPBs has been widely studied. Aggson et al. (1996) used observations from satellites to determine that appearances of BS in EPBs are related to the electric field arising from the plasma depletion region. Some studies have suggested that occurrences of BSEPB are due to the polarization electric fields associated with EPBs (Li GZ et al., 2011; Wu K et al., 2017). Makela et al. (2006) presented evidence that BSEPBs are caused by secondary instabilities. A number of simulations have also been performed to study the evolution of BSEPBs. Zalesak et al. (1982) used a three-layer model to simulate a simple BSEPB under a certain diffusion parameter and a ratio of conductivity relative to the background. Huba and Joyce (2008) showed that a 2D simulation code (NRLESF2) could generate multiple BSEPBs. Yokoyama et al. (2014) reproduced the nonlinear growth of EPB and BSEPB process, using a three-dimensional high-resolution bubble model. Although the evolution of BSEPBs has been widely studied and simulated, their occurrence characteristics have not been investigated in detail. 
In this paper, we used seven-years (2012-2018) of observations from two all-sky imagers located at Fuke and Qujing, China to study BSEPB occurrence characteristics. Statistical results show that $B S$ occurs with a high incidence in the evolution of EPBs. However, not all EPBs are accompanied by BS occurrence. Occurrence times of BSEPBs change with local time. Furthermore, their occurrence characteristics are found to be related to solar activity and geomagnetic activity.

\section{Instrumentation}

The airglow data used in this study were from two all-sky imagers, which were deployed at low-latitude regions in southern China. One of them operates over Fuke, China (Geographic: $19.5^{\circ} \mathrm{N}$, 109.1 $1^{\circ}$; Geomagnetic: $9.5^{\circ} \mathrm{N}, 178.4^{\circ} \mathrm{W}$ ); the other operates over Qujing, China (Geographic: $25^{\circ} \mathrm{N}, 104^{\circ} \mathrm{E}$; Geomagnetic: $15.1^{\circ} \mathrm{N}$, $\left.176.0^{\circ} \mathrm{E}\right)$. Their locations are indicated by the stars in Figure 1. The imagers have the same design. Each all-sky imager consists primarily of a fish-eye lens with a $180^{\circ}$ field of view, a CCD detector (1024 × 1024 pixel), and an interference filter $(630.0 \mathrm{~nm})$. The integration time is $3 \mathrm{~min}$. An introduction to the two all-sky imagers has been given in several previous publications (e.g., Wu K et al., 2018, 2020, 2021).

\section{Observations and Results}

In Figure 2, we present some typical examples of EPBs with BS. A red line denotes the main stem of an EPB and a yellow line denotes BS on an EPB. Images of Figure 2 are from two all-sky imagers. For these images, we used an unwarping (Garcia et al., 1997) process to remove the effects of compression and curing by the all-sky imagers. These images have been mapped into a corresponding geographic range; in this process, the airglow layer

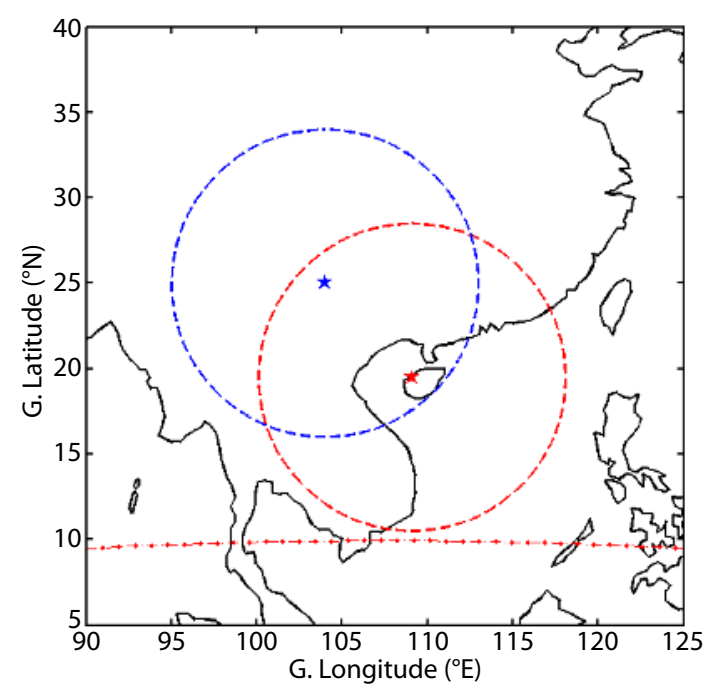

Figure 1. The locations of the two all-sky imagers. The stars denote the geographic locations of the all-sky imagers at Qujing and Fuke. The blue and red circles denote the fields of view, respectively of the two imagers at an altitude of $250 \mathrm{~km}$. The red dotted line represents the magnetic equator.

was assumed to be at $250 \mathrm{~km}$. Figure 2 shows EPBs associated with various BSs, such as no BS (a), two BSs (b, c), three BSs (d), and multi-BS (e, $f$ ) (more than three branches). It is generally true that most EPBs display one or more occurrences of BS in their development. These BSs are related to the morphological characteristics and evolutions of the EPBs. Unfortunately, occurrence characteristics of BSEPBs have not previously been well addressed.

In order to study occurrence characteristics of BSEPBs, we used

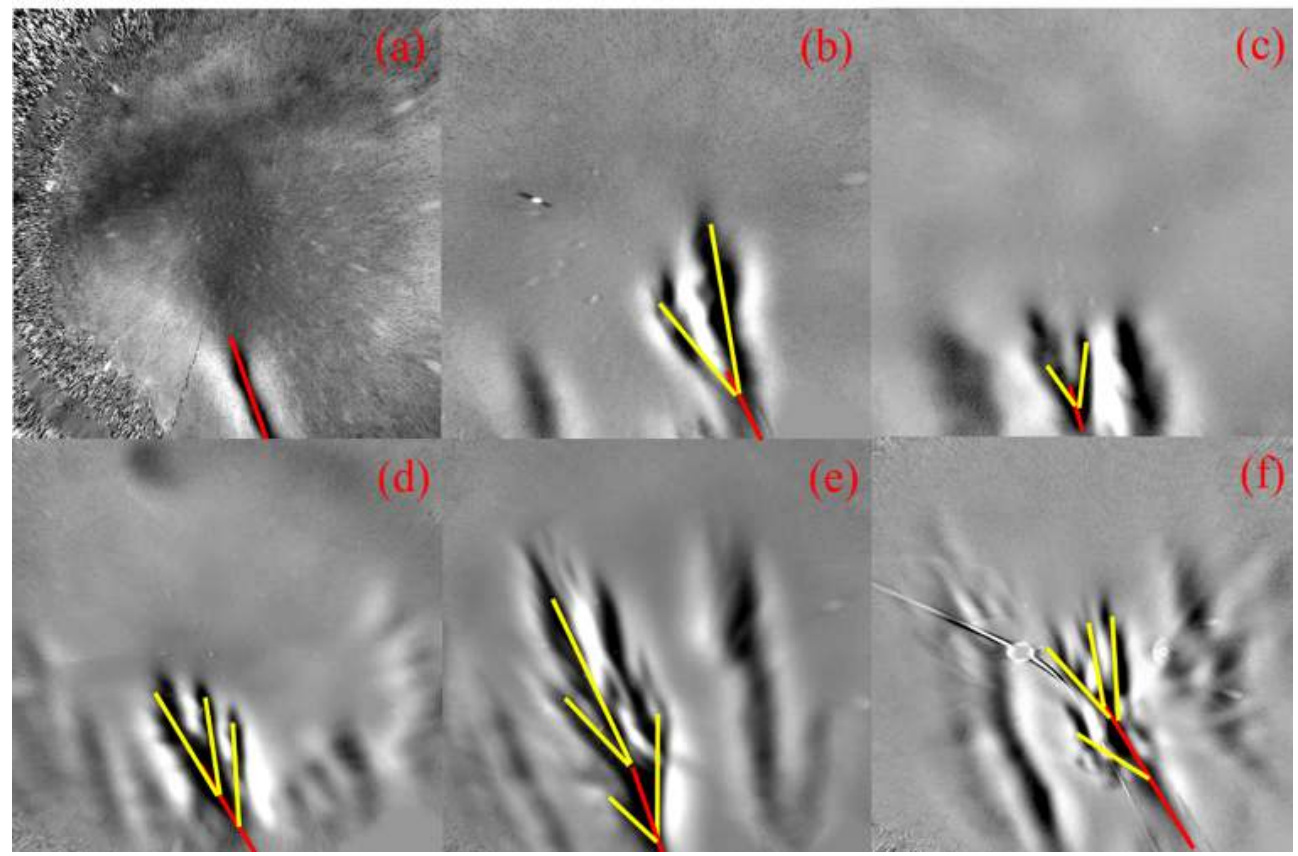

Figure 2. Various BSs of images were obtained from two all-sky imagers that were located at Qujing and Fuke, China. (a) no BS; (b) and (c) two BSs; (d) three BSs; (e) and (f) multi-BSs. These images were mapped to geographical coordinates; in the image processing, the airglow layer was assumed to be at $250 \mathrm{~km}$. 
2012-2018 (seven years) of observation data from the two stations. We investigated every EPB event for evidence of BS to determine BS incidence rate. During the statistical process, we considered only branching of the main stem; we did not count subbranching (branches of branches). Table 1 gives data categories of two all-sky imagers, including year, total EPB days, no BS EPB days, days on which some EPBs exhibited BS, and days on which all EPBs were observed to branch. Numbers in brackets represent the subset of geomagnetically disturbed days (if Dst index is $<-30 \mathrm{nT}$ before all EPBs disappear, we defined it is a geomagnetically disturbed day) within each category. Figure 3 shows occurrence characteristics derived from data collected by the two all-sky imagers. In Figure 3, the black column presents total EPB days; the gray column indicates EPB days on which no branching was observed; the green column presents days on which BS was observed in some EPBs; the red column indicates days when all EPBs had BS; the white column gives the subsets of geomagnetically disturbed days.

These data presented in Figure 3 reveal some significant occurrence characteristics of BSEPB. It can be seen that BS appeared on most days when EPBs were observed (in every year, the values in the gray column are much less than the ones green column plus red column). In other words, BS is a high occurrence phenomenon during the evolution of EPBs. On most days when EPBs were observed, at least one of them exhibited branching; however, it is worth noting that not all EPBs were observed to branch. Also worthy of note is that in every year the numbers in the green column are relatively larger than those in the red column; in other words, days on which all EPBs exhibit branching structure are rel-

Table 1. BS occurrence observed by all-sky imagers at Qujing and Fuke.

\begin{tabular}{cccccc}
\hline Year & $\begin{array}{c}\text { Total } \\
\text { EPB days }\end{array}$ & $\begin{array}{c}\text { Days } \\
\text { when no } \\
\text { EPBs } \\
\text { exhibit BS }\end{array}$ & $\begin{array}{c}\text { Days when } \\
\text { some EPBs } \\
\text { exhibit BS }\end{array}$ & $\begin{array}{c}\text { Days } \\
\text { when all } \\
\text { EPBs } \\
\text { exhibit BS }\end{array}$ & Station \\
\hline 2012 & $20(11)$ & $3(2)$ & $17(9)$ & 0 & \\
2013 & $24(3)$ & $3(0)$ & $19(3)$ & $2(0)$ & \\
2014 & $56(6)$ & $3(1)$ & $37(5)$ & $16(0)$ & \\
2015 & $21(6)$ & $3(1)$ & $16(5)$ & $2(0)$ & Qujing \\
2016 & $8(4)$ & $2(2)$ & $6(2)$ & 0 & \\
2017 & 0 & 0 & 0 & 0 & \\
2018 & $1(0)$ & $1(0)$ & 0 & 0 & \\
\hline 2012 & 0 & 0 & 0 & 0 & \\
2013 & $46(8)$ & $7(1)$ & $34(6)$ & $5(1)$ & \\
2014 & $92(31)$ & $5(2)$ & $52(24)$ & $35(5)$ & \\
2015 & $39(19)$ & $6(4)$ & $30(15)$ & $3(0)$ & Fuke \\
2016 & $25(7)$ & $4(1)$ & $19(6)$ & $2(0)$ & \\
2017 & $11(2)$ & $2(0)$ & $8(2)$ & $1(0)$ & \\
2018 & $12(5)$ & $5(1)$ & $7(4)$ & 0 & \\
\hline
\end{tabular}

Note: $\mathrm{BS}=$ branching structure; $\mathrm{EPB}=$ equatorial plasma bubble. Numbers in brackets represent the subset of geomagnetically disturbed days in each category. atively rare though not as rare as days on which no branching of EPBs is observed; in other words, on most EPB days, some EPBs exhibit BS but others do not. The data in Figure 3 also reveal another interesting fact - that the frequency of occurrence of branching EPBs appears to be linked to solar activity. During the solar maximum (2014 in our data set), days of EPB with BS increase significantly. In particular, the values in the red column increased significantly; that is, an increased incidence of EPBs with branching structure was observed, clearly related to solar activity; the incidence of branching EPBs was highest during the solar maximum. Note also that numbers in the white column correlate strongly with those in the BS column, which means that on days when geomagnetic disturbances were observed, most EPBs exhibited BS, strongly suggesting a link between geomagnetic activity and the occurrence of BSEPBs.

Another variable of interest to the study of occurrence characteristics of BSEPB is the occurrence time of the first BS in every BSEPB case. These data are given in Table 2, which presents year, efficient days (except for the days without branching EPBs and days in which occurrence time of first BS cannot be determined), and occurrence times of first BSEPB. These results are displayed in Figure 4: the black column presents efficient days; the gray column presents days on which the first BS appeared between 20:00 and 21:00 LT; the green column presents days on which the
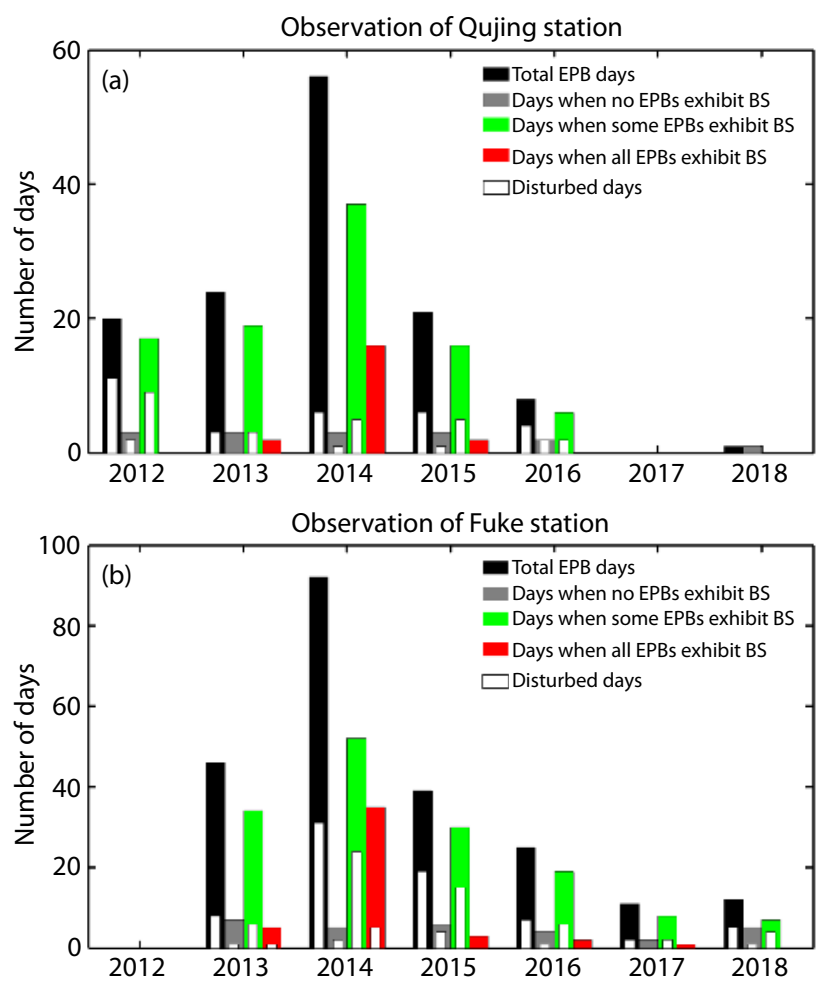

Figure 3. Statistical results of BS occurrences observed by all-sky imagers in Qujing (a) and Fuke (b), China during years 2012 to 2018. The black column presents total EPB days; the gray column presents the number of EPB days during which no branching was detected; the green column presents EPB days on which at least some EPBs exhibited BS; the red column presents days on which all observed EPBs exhibited BS; the white column presents geomagnetically disturbed days. 
Table 2. Occurrence time of BS varied with local time at Qujing and Fuke.

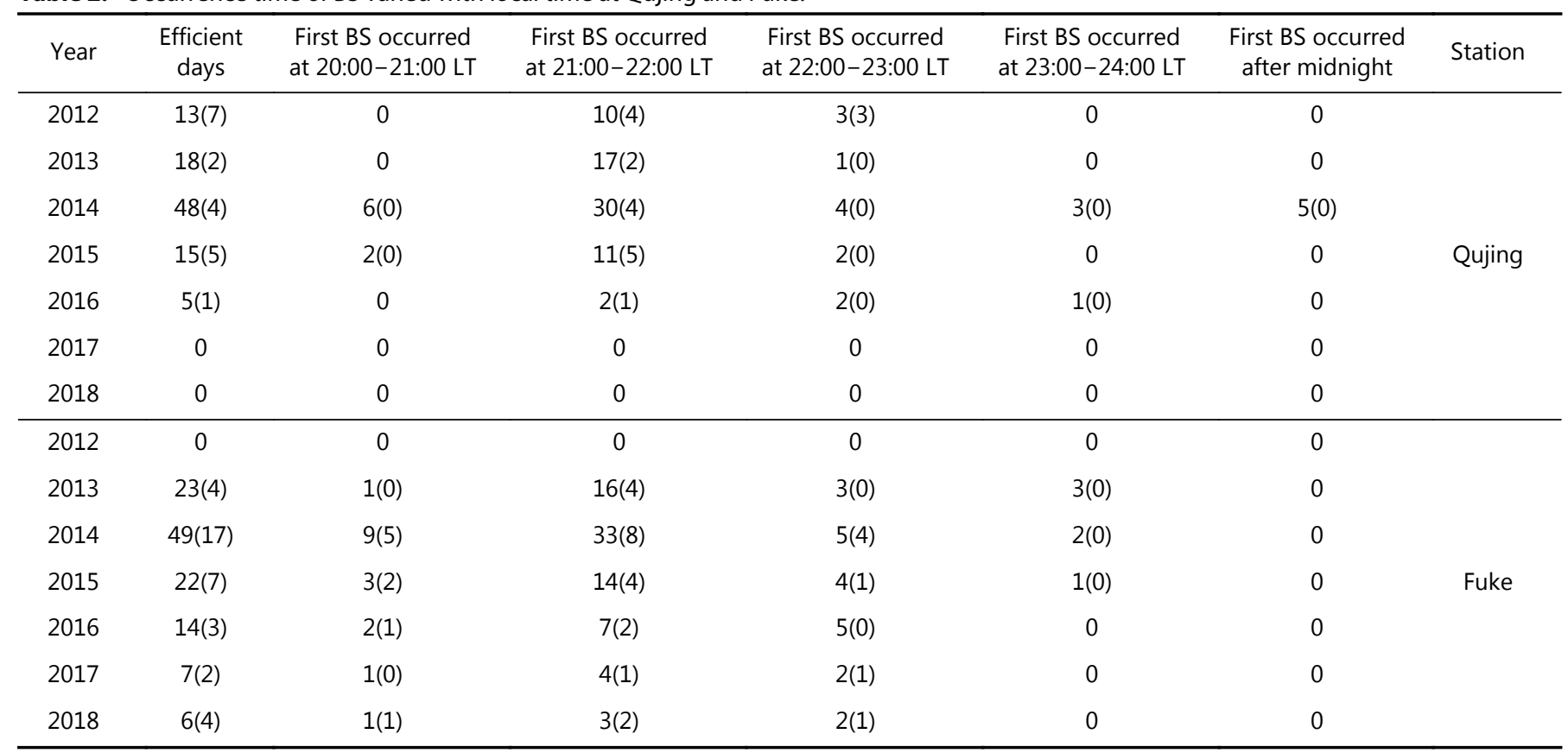

Note: $\mathrm{BS}=$ branching structure; $\mathrm{EPB}=$ equatorial plasma bubble. Numbers in brackets represent the subset of geomagnetically disturbed days in each category.

first BS appeared between 21:00 and 22:00 LT; the blue column records the days when first BS appeared between 22:00 and 23:00 LT; appearances between 23:00 and 24:00 LT are presented in the red column; the yellow column indicates days on which the first BS appeared after midnight; the white column within each column records days on which geomagnetic-disturbance was detected. Examination of the data in Figure 4 reveals that the appearance times of BSEPBs varied with local time. For branching EPBs, most of the first instances of BS appeared between 21:00 and 22:00 LT. Others often appeared between 22:00 and 24:00 LT. Interestingly, appearances of the first BS after midnight began to occur during the solar maximum (for example 2014). Note also that comparison of days with and without geomagnetic disturbances reveals no correlation with appearance time of BS; thus we find no obvious link between geomagnetic activity and BS appearance time.

To investigate further the occurrence characteristics of BSEPB, we used statistics to investigate the significance of various structural forms of BS. Detailed data are shown in Table 3, which displays, for each year, total BS days (EPB days on which no branching was observed are not included), two-BS days, three-BS days, multi-BS days, and days when various BSs were observed to exist simultaneously. Figure 5 presents the statistical result of these different BSs. In Figure 5, the black column presents total BS days; the gray column, days when two-branch EPBs were observed; the green column, three-branch EPB days; the blue column, days on which branching higher than three was observed; the red column, days on which BSs with different structures were observed to exist simultaneously; the white column in each parent column records the subset of days on which geomagnetic disturbances were observed. Figure 5 reveals some significant statistical results. Certain BS types occurred more frequently than others. When branching was observed, most were two- or three-branch structures. Structures with more than three branches ("multi-branch") appeared only in 2014, the year of highest solar activity. Moreover, Figure 5 shows that days on which various forms of BSEPB were observed to coexist increased from 2012 to 2014 and decreased from 2014 to 2018. There was also a positive correlation between BSEPB count and solar flux; thus, BSEPB occurrence appears to be related to solar activity.

\section{Discussion}

In Section 3, occurrence characteristics of BSEPB were investigated in detail. Statistical results of seven-years (2012-2018) of observations from two all-sky imagers reveal that BS appears to be a high occurrence phenomenon in the evolution of EPBs. However, not every EPB exhibited BS during its evolution. Meanwhile, this analysis demonstrates that occurrence times of BSEPBs varied with local time, and that their occurrence characteristics were related to solar activity. These results will enhance our understanding of EPB evolution.

BS is an important morphological feature of EPBs. By studying the occurrence of BS, we can better understand the stability and complexity of EPBs. Occurrence of BS should be an important reflection of EPB stability. It is generally accepted that Rayleigh-Taylor instability is the most plausible mechanism triggering EPBs. However, the evolution of EPBs will be affected by other factors, such as secondary instabilities known to be associated with EPBs. Many studies have found that EPBs usually are influenced by secondary instabilities along the EPB wall (Makela et al., 2006; Yokoyama et al., 2014). Secondary instabilities will cause BS to appear in the EPB. Secondary instabilities usually occur on the western wall of an EPB, which can promote the develop of a BS (Tsunoda et al., 1982). Makela et al. (2006) reported that secondary instabilities 

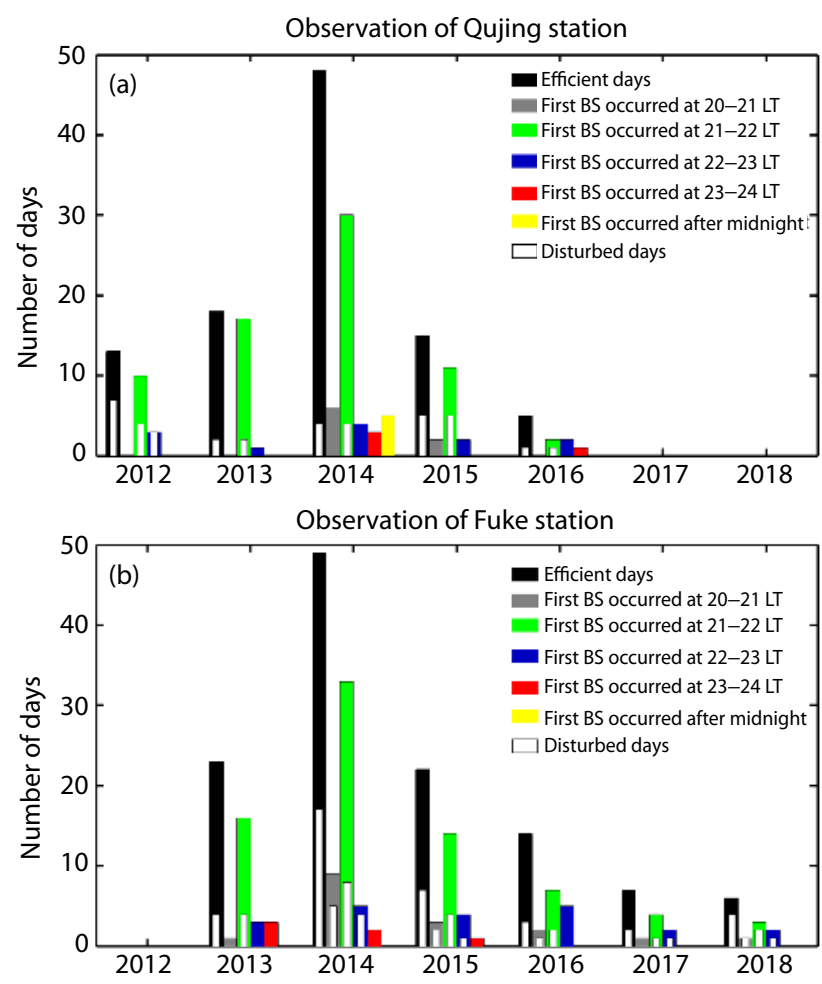

Figure 4. Occurrence time of branching structure (years 2012 to 2018) varied with local time. (a) and (b) show statistical results obtained from Qujing station and Fuke station, respectively. The black column presents the efficient days (except for days without branching EPBs and days on which occurrence time of first BS cannot be determined); the gray column presents days on which the first BS appeared between 20:00 and 21:00 LT; the green column presents days when first BS appeared between 21:00 and 22:00 LT; the blue column records days when first BS appeared between 22:00 and 23:00 $\mathrm{LT}$; days in the red column are those on which the time of first BS was between 23:00 and 24:00 LT; the yellow column gives days on which the time of first BS was after midnight; the white portion of every column records the subset of days on which geomagneticdisturbance was observed.

developed in the unstable wall of EPBs and caused BSs to develop. Huba et al. (2008) used a 2-D simulation code to show that multiple BS can be caused by secondary instabilities. Yokoyama et al. (2014) used a three-dimensional high-resolution numerical model to produce BS processes caused by secondary instabilities, suggesting that secondary instabilities play an important role in the development of BSEPBs. Growth of secondary instabilities was affected by many factors, such as magnetic field, electric field, and density gradient (Kelley, 2009). Although many simulations have produced BSs by using secondary instabilities, it is difficult to measure empirically these secondary instabilities associated with EPBs. Moreover, in these simulations, secondary instabilities associated with EPB were a main reason for triggering the BS. We thus propose to use the characteristics of BS to study the complexity and stability of an EPB case; in other words, the occurrence of BS can be used to predict the intensity of the hard-to-observe secondary instabilities associated with EPBs and the stability strength of EPBs, which means that it is important to understand the occur-
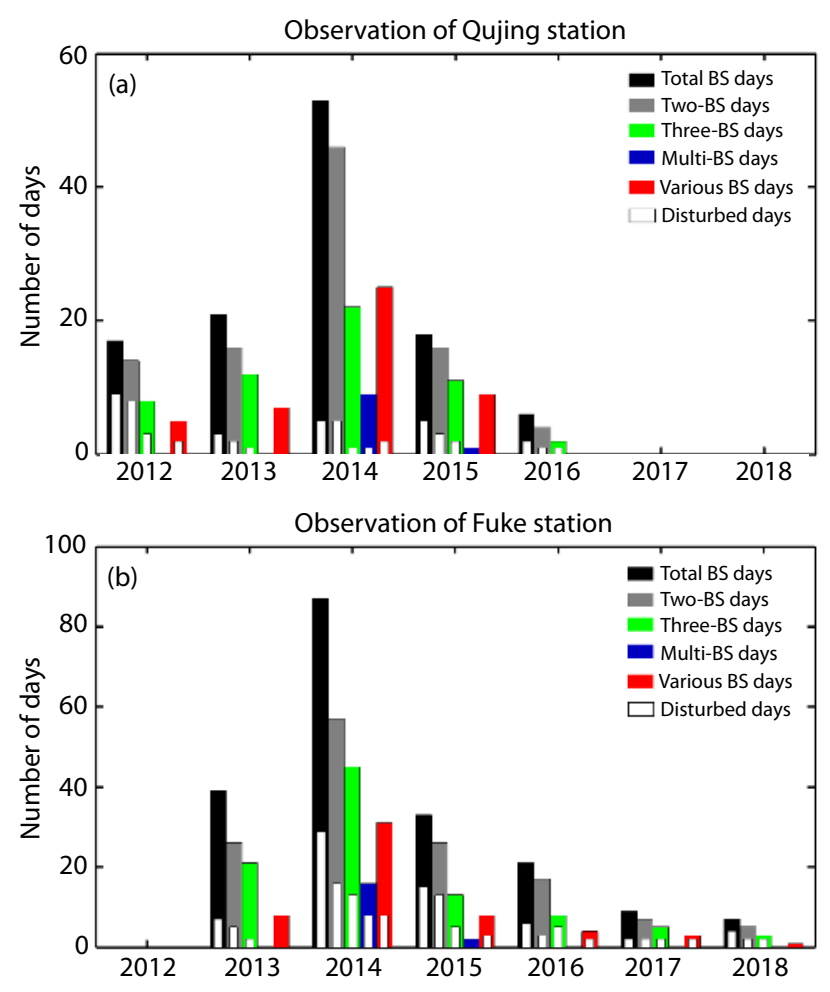

Figure 5. Statistical results of branching structure were obtained from Qujing station (a) and Fuke station (b). The black column presents total branching days; the gray, days of two-branch EPBs; the green column, days of three-branch EPBs; the blue column, days of multi-branch EPBs; the red column, days on which various branching structure existed simultaneously; the white portion of every column presents the subset of days on which geomagnetic disturbance was detected.

\section{rence characteristics of BSEPBs.}

In addition, the relationship between BSEPBs and solar activity is an issue worthy of discussion. Based on the statistical results presented in Section 3, solar activity appears to affect the occurrence characteristic of BSEPBs. A number of studies have found that solar activity can affect EPB occurrence (Tsunoda, 1985; Su SY et al., 2006, 2007). Incidence rates of EPBs usually change with solar activity: there is higher incidence of EPBs during high solar activity years (Huang CY et al., 2002; Stolle et al., 2006). But the relationship between BSEPBs and solar activity has not been well understood. Our study indicates that the occurrence of BS is related to solar activity. An obvious increase in total days of all EPBs with BS was observed in 2014, the year in which solar cycle 24 reached its maximum. Moreover, that proportion had been rising each year from 2012 to 2014, and fell from its 2014 peak in 2015 and again in 2016. The occurrences of BS varieties were similar to the year varieties of solar flux. In other words, occurrence of various BSEPB structures appear to be affected by solar activity. High solar activity can effect the ionosphere environment, thereby contributing to occurrences of BSEPB. During high solar activity, the bottom altitude of the ionosphere usually is higher than when solar activity is lower (Vichare and Richmond, 2005; Fejer et al., 2008a; Stolle et al., 2008; Su SY et al., 2008). Solar activity favors the growth of instabilities, which promote the occurrence of 
Table 3. BS types were obtained from Qujing and Fuke.

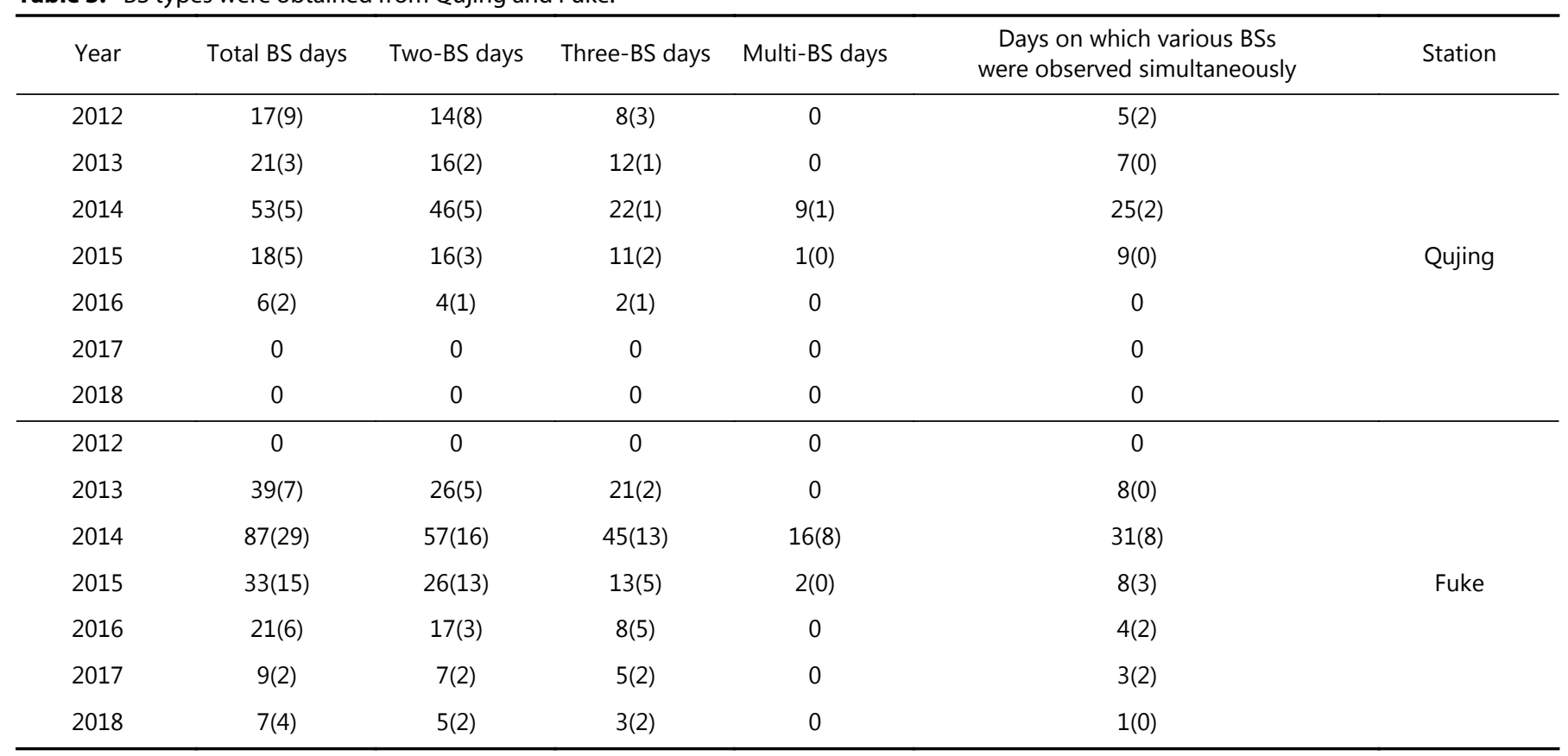

Note: $\mathrm{BS}=$ branching structure; $\mathrm{EPB}=$ equatorial plasma bubble. Numbers in brackets represent the subset of geomagnetically disturbed days in each category.

BSEPBs. Since the appearance of BS is a common part of EPB evolution, the factors beneficial to occurrence of EPBs are also advantageous for increased BS inducement. Significantly more vertical drifts of the $F$ region are observed in years of high solar activity than in years of lower solar activity (Fejer et al., 1979; Batista et al., 1996; Abdu et al., 2006). Many studies have shown that the occurrence of EPBs is linearly correlated with nighttime equatorial upward plasma drift (Vichare and Richmond, 2005; Fejer et al., 2008a). Stronger upward plasma drift during a solar maximum should cause a higher incidence rate of BSEPBs. Besides, due to larger electron densities and temperatures, instability is more likely to occur in those EPBs, which suggests that EPBs should be more prone to a complex structure during solar maximums; that is, we can expect a higher incidence rate of BSEPB. Various BS forms of EPB indicate the complexity of EPB. It is a highly complex EPB case when there are two or more branches (three-branch structure and multi-branch - more than three branches). The statistical results presented in Figure 5 showed that complex EPB events were related to solar activity; note that the multi-branch structure appeared only in the year of highest solar activity, and that the days of coexistence of various BSEPB structures increased obviously during that year (2014). These results also indicate a relationship between BSEPB and solar activity.

As for evidence of a relationship between occurrence of BSEPBs and local time, Figure 4 demonstrates that the BSEPB occurrence changed with local time. The first BSEPB most often occurred after 20:00 LT but it could occur at any time during the lifetime of a given EPB. The largest number of first appearances of branching was observed between 21:00 and 22:00 LT, the frequency of occurrence gradually decreasing after 22:00 LT except for the year 2014. This result is similar to the pattern of occurrence characteristic of EPBs versus local time. Stolle et al. (2006) report, using CHAMP data from 2001-2004, that the highest occurrence of EPBs in those years appeared between 21:00 and 22:00 LT. Xiong $C$ et al. (2010), using data from CHAMP and GRACE, also found an EPB occurrence peak between 21:00 and 22:00 LT. In summary, observations of EPBs and of EPBS with branching structure both peak in the same time interval. Interestingly, instances of first BSEPB after midnight were observed primarily during the high solar activity year, 2014, suggesting that high solar activity may restrain the starting time of BS in some EPBs.

An important aspect of BS is the relationship between occurrence of BSEPB and geomagnetic activity. Some previous studies have reported that the occurrence of EPBs is affected by geomagnetic activity (e.g., Abdu et al., 2003; Keskinen et al., 2006; Sahai et al., 2009; Patra et al., 2016; Aa et al., 2018). Geomagnetic storms can cause equatorial ionospheric variations by Prompt Penetrating Electric Fields (PPEF) and Disturbance Dynamo Electric Fields (DDEF), which are supposed to cause EPBs (Kelley et al., 1979; Richmond et al., 2003). Results presented in Figure 3 confirm that BS was observed on most EPB days characterized by strong geomagnetic disturbances. Occurrence of BSEPBs can be expected, also, to correlate with geomagnetic activity because BS is a common feature of the evolution of EPBs. Table 4 and Figure 6 illustrate this observation. In storm time, increases in the hourly $A E$ indices and long-lasting $A E$ indices can be used to indicate PPEF and DDEF (Ahn et al., 1992; Fejer et al., 2008b; Kelley, 2009). Using the criterion from Fejer and Scherliess (1995), PPEF or DDEF may occur when an hourly variation of $A E$ indices is greater than $125 \mathrm{nT}$, or a four hours' average is greater than $300 \mathrm{nT}$ on a geomagnetic disturbed day. When $A E$ indices satisfy the above criterion during the evolution of an EPB, we defined the disturbed day as a disturbed AE day. In Figure 6, EPBs show BS on most of the disturbed $A E$ days, indicating that PPEF or DDEF caused by the geomagnet- 
Table 4. BS occurrence in disturbed AE days.

\begin{tabular}{ccccc}
\hline Year & Total disturbed days with EPB & Total disturbed days without BS & Total disturbed days with BS & Station \\
\hline $2012-2018$ & $30(25)$ & $6(2)$ & $24(23)$ & Qujing \\
$2012-2018$ & $72(62)$ & $9(2)$ & $63(60)$ & Fuke \\
\hline
\end{tabular}

Note: $\mathrm{BS}=$ branching structure; $\mathrm{EPB}=$ equatorial plasma bubble. Numbers in brackets represent geomagnetically disturbed $\mathrm{AE}$ days corresponding to each category.
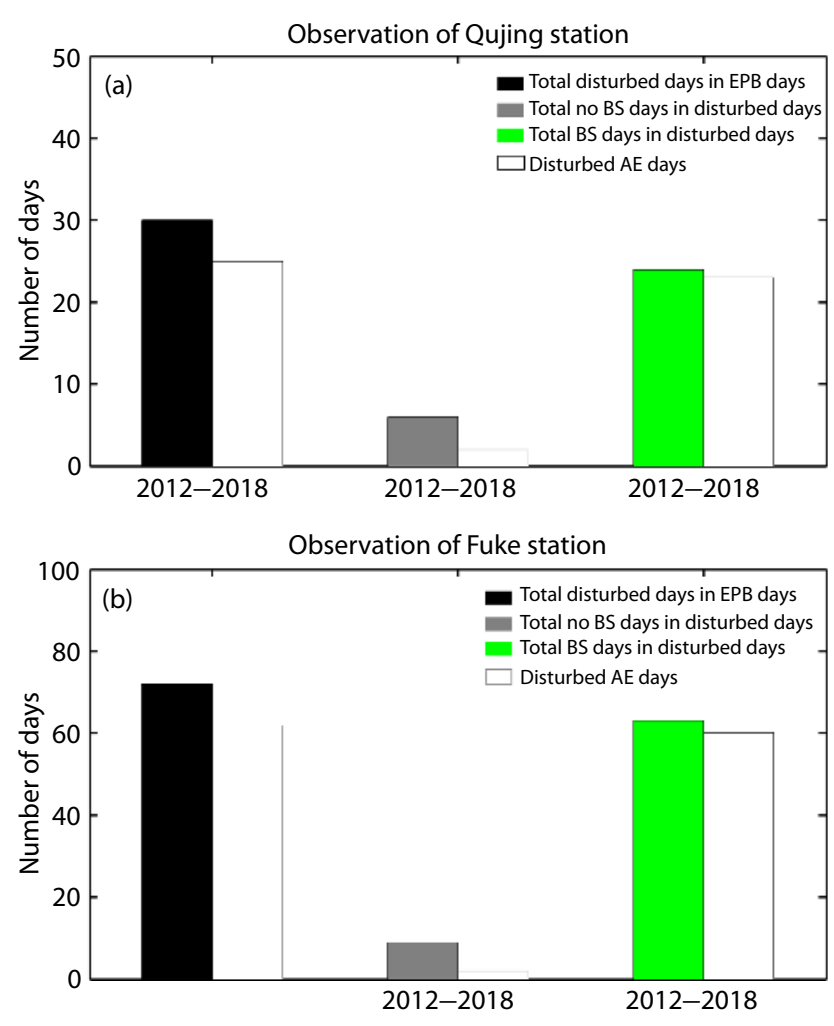

Figure 6. Statistical results of BS occurrence in disturbed AE days at Qujing station (a) and Fuke station (b). The black column presents total disturbed days with EPB; the gray, total disturbed days without $\mathrm{BS}$; the green column, total disturbed days with BS; the white column, the subset of total disturbed AE days in each category.

ic activity affects the occurrence frequency of BSEPB.

Occurrence of BSEPB should be affected by disturbance winds and westward winds during geomagnetic disturbances. During disturbance geomagnetic activity, the winds are observed to be mainly westward at low latitudes (Xiong $C$ et al., 2015). When the wind blows westward, the wind-induced Pedersen current flows downward, and gradient-drift instability can occur at the eastern wall of EPB, where the plasma density gradient is eastward. Meanwhile, due to the Coriolis force, the enhanced equatorward wind at disturbed periods also will have a westward component that will work on the eastward wall of EPB, causing secondary instabilities. Similar findings of BSEPB from secondary instabilities have been reported by Makela et al. (2006), instabilities caused by disturbance winds and westward winds associated with increased geomagnetic activity.

\section{Summary}

In this study, we used seven-years (2012-2018) of observations from two all-sky imagers to investigate detailed occurrence characteristics of branching structures of EPB. The main findings can be summarized as follows:

(1) Branching structure is a high occurrence phenomenon during the evolution of EPBs; most are observed to branch. However, not all EPBs are observed to develop branching.

(2) Occurrence characteristics of branching structures are related to solar activity. The number of EPB days on which EPB branching was observed increased significantly during high solar activity (for example, in 2014), especially the number of days on which all EPBs were observed to exhibit branching.

(3) Time of branching occurrence varied with local time. Also, although within the aggregated data for the entire seven-year period the most common time interval in which a day's first branching structure appeared was 21:00 to 22:00 LT, during the solar maximum year (2014), first EPB branching structures were observed to be delayed on some days until after midnight.

(4) In some EPBs, more than one form of branching structure was observed to occur in the course the EPB's evolution, such as twobranch structure, three-branch structure and multi-branch (more than three branches) structure. Two-branch and three-branch structures appeared most frequently; multi-branch structure appeared only in the high solar activity year, during which instances of branching structure coexistence were also observed to increase significantly.

(5) Branching structure may be related to EPB stability. During high solar activity, there is poor stability in EPBs, especially between 21:00-22:00 LT.

\section{Acknowledgments}

We acknowledge the use of data from the Chinese Meridian Project. This work was supported by the Youth Science and Technology Innovation Foundation of NSSC, the International Partnership Program of Chinese Academy of Sciences (183311KYSB20200003), the National Natural Science Foundation of China (41831073 and 42004138), and the Open Research Project of Large Research Infrastructures of CAS - "Study on the interaction between low/mid-latitude atmosphere and ionosphere based on the Chinese Meridian Project". The airglow data were downloaded from http://data.meridianproject.ac.cn/. The Dst, Kp, and $F_{10.7}$ data were obtained from the OMNIWeb (https://omniweb.gsfc.nasa.gov/).

\section{References}

Aa, E., Huang, W. G., Liu, S. Q., Ridley, A., Zou, S. S., Shi, L. Q., Chen, Y. H., Shen, H., Yuan, T. J., ... Wang. T. (2018). Midlatitude plasma bubbles over China and adjacent areas during a magnetic storm on 8 September 2017. Space Wea., 16(3), 321-331. https://doi.org/10.1002/2017SW001776

Abdu, M. A., Batista, I. S., Takahashi, H., MacDougall, J., Sobral, J. H., Medeiros, A. F., and Trivedi, N. B. (2003). Magnetospheric disturbance induced equatorial 
plasma bubble development and dynamics: a case study in Brazilian sector. J. Geophys. Res.: Space Phys., 108(A12), 1449.

https://doi.org/10.1029/2002ja009721

Abdu, M. A., Batista, I. S., Reinisch, B. W., Sobral, J. H. A., and Carrasco, A. J. (2006). Equatorial $F$ region evening vertical drift, and peak height, during southern winter months: a comparison of observational data with the IRI descriptions. Adv. Space Res., 37(5), 1007-1017.

https://doi.org/10.1016/j.asr.2005.06.074

Aggson, T. L., Laakso, H., Maynard, N. C., and Pfaff, R. F. (1996). In situ observations of bifurcation of equatorial ionospheric plasma depletions. J. Geophys. Res.: Space Phys., 101(A3), 5125-5132. https://doi.org/10.1029/95ja03837

Ahn, B. H., Kamide, Y., Kroehl, H. W., and Gorney, D. J. (1992). Cross-polar cap potential difference, auroral electrojet indices, and solar wind parameters. J. Geophys. Res.: Space Phys., 97(A2), 1345-1352.

https://doi.org/10.1029/91JA02432

Batista, I. S., de Medeiros, R. T., Abdu, M. A., de Souza, J. R., Bailey, G. J., and de Paula, E. R. (1996). Equatorial ionospheric vertical plasma drift model over the Brazilian region. J. Geophys. Res.: Space Phys., 101(A5), 10887-10892. https://doi.org/10.1029/95ja03833

Fejer, B. G., Farley, D. T., Woodman, R. F., and Calderon, C. (1979). Dependence of equatorial $F$ region vertical drifts on season and solar cycle. J. Geophys. Res.: Space Phys., 84(A10), 5792-5796. https://doi.org/10.1029/JA084iA10p05792

Fejer, B. G., and Scherliess, L. (1995). Time dependent response of equatorial ionospheric electric fields to magnetospheric disturbances. Geophys. Res. Lett., 22(7), 851-854. https://doi.org/10.1029/95GL00390

Fejer, B. G., Jensen, J. W., and Su, S. Y. (2008a). Quiet time equatorial $F$ region vertical plasma drift model derived from ROCSAT-1 observations. J. Geophys. Res.: Space Phys., 113(A5), A05304. https://doi.org/10.1029/2007JA012801

Fejer, B. G., Jensen, J. W., and Su, S. Y. (2008b). Seasonal and longitudinal dependence of equatorial disturbance vertical plasma drifts. Geophys. Res. Lett., 35(20), L20106. https://doi.org/10.1029/2008GL035584

Garcia, F. J., Taylor, M. J., and Kelley, M. C. (1997). Two-dimensional spectral analysis of mesospheric airglow image data. Appl. Opt., 36(29), 7374-7385. https://doi.org/10.1364/ao.36.007374

Huang, C. Y., Burke, W. J., Machuzak, J. S., Gentile, L. C., and Sultan, P. J. (2002). Equatorial plasma bubbles observed by DMSP satellites during a full solar cycle: toward a global climatology. J. Geophys. Res.: Space Phys., 107(A12), 1434. https://doi.org/10.1029/2002ja009452

Huba, J. D., Joyce, G., and Krall, J. (2008). Three-dimensional equatorial spread $F$ modeling. Geophys. Res. Lett., 35(10), L10102. https://doi.org/10.1029/2008GL033509

Kelley, M. C., Fejer, B. G., and Gonzales, C. A. (1979). An explanation for anomalous equatorial ionospheric electric fields associated with a northward turning of the interplanetary magnetic field. Geophys. Res. Lett., 6(4), 301-304. https://doi.org/10.1029/GL006i004p00301

Kelley, M. C. (2009). The Earth's lonosphere: Plasma Physics and Electrodynamics (2nd ed). San Diego: Academic Press.

Keskinen, M. J., Ossakow, S. L., Fejer, B. G., and Emmert, J. (2006). Evolution of equatorial ionospheric bubbles during a large auroral electrojet index increase in the recovery phase of a magnetic storm. J. Geophys. Res.: Space Phys., 111(A2), A02303. https://doi.org/10.1029/2005ja011352

Li, G. Z., Ning, B. Q., Patra, A. K., Wan, W. X., and Hu, L. H. (2011). Investigation of low-latitude $E$ and valley region irregularities: their relationship to equatorial plasma bubble bifurcation. J. Geophys. Res.: Space Phys., 116(A11), A11319. https://doi.org/10.1029/2011JA016895

Makela, J. J., Kelley, M. C., and Nicolls, M. J. (2006). Optical observations of the development of secondary instabilities on the eastern wall of an equatorial plasma bubble. J. Geophys. Res.: Space Phys., 111 (A9), A09311. https://doi.org/10.1029/2006JA011646

Makela, J. J., and Otsuka, Y. (2012). Overview of nighttime ionospheric instabilities at low-and mid-latitudes: coupling aspects resulting in structuring at the mesoscale. Space Sci. Rev., 168(1-4), 419-440. https://doi.org/10.1007/s11214-011-9816-6

Mendillo, M., and Baumgardner, J. (1982). Airglow characteristics of equatorial plasma depletions. J. Geophys. Res.: Space Phys., 87(A9), 7641-7652. https://doi.org/10.1029/JA087iA09p07641

Mendillo, M., and Tyler, A. (1983). Geometry of depleted plasma regions in the equatorial ionosphere. J. Geophys. Res.: Space Phys., 88(A7), 5778-5782. https://doi.org/10.1029/JA088iA07p05778

Ossakow, S. L. (1981). Spread-F theories-a review. J. Atmos. Terr. Phys., 43(5-6), 437-452. https://doi.org/10.1016/0021-9169(81)90107-0

Ott, E. (1978). Theory of Rayleigh-Taylor bubbles in the equatorial ionosphere. J. Geophys. Res.: Space Phys., 83(A5), 2066-2070. https://doi.org/10.1029/ja083ia05p02066

Patra, A. K., Chaitanya, P. P., Dashora, N., Sivakandan, M., and Taori, A. (2016). Highly localized unique electrodynamics and plasma irregularities linked with the 17 March 2015 severe magnetic storm observed using multitechnique common-volume observations from Gadanki, India. J. Geophys. Res.: Space Phys., 121(11), 11518-11527. https://doi.org/10.1002/2016JA023384

Richmond, D. A., Peymirat, C., and Roble, R. G. (2003). Long-lasting disturbances in the equatorial ionospheric electric field simulated with a coupled magnetosphere-ionosphere-thermosphere model. J. Geophys. Res.: Space Phys., 108(A3), 1118. https://doi.org/10.1029/2002JA009758

Sahai, Y., Becker-Guedes, F., Fagundes, P. R., de Jesus, R., de Abreu, A. J., Otsuka, Y., Shiokawa, K., Igarashi, K., Yumoto, K., ... Bittencourt, J. A. (2009). Effects observed in the ionospheric $F$ region in the east Asian sector during the intense geomagnetic disturbances in the early part of November 2004. J. Geophys. Res.: Space Phys., 114(A3), A00A18. https://doi.org/10.1029/2008JA013053

Stolle, C., Lühr, H., Rother, M., and Balasis, G. (2006). Magnetic signatures of equatorial spread $F$ as observed by the CHAMP satellite. J. Geophys. Res.: Space Phys., 111(A2), A02304. https://doi.org/10.1029/2005JA011184

Stolle, C., Lühr, H., and Fejer, B. G. (2008). Relation between the occurrence rate of ESF and the equatorial vertical plasma drift velocity at sunset derived from global observations. Ann. Geophys., 26(12), 3979-3988. https://doi.org/10.5194/angeo-26-3979-2008

Su, S. Y., Liu, C. H., Ho, H. H., and Chao, C. K. (2006). Distribution characteristics of topside ionospheric density irregularities: equatorial versus midlatitude regions. J. Geophys. Res.: Space Phys., 111(A6), A06305. https://doi.org/10.1029/2005JA011330

Su, S. Y., Chao, C. K., Liu, C. H., and Ho, H. H. (2007). Meridional wind effect on anti-solar activity correlation of equatorial density irregularity distribution. J. Geophys. Res.: Space Phys., 112(A10), A10305. https://doi.org/10.1029/2007ja012261

Su, S. Y., Chao, C. K., and Liu, C. H. (2008). On monthly/seasonal/longitudinal variations of equatorial irregularity occurrences and their relationship with the postsunset vertical drift velocities. J. Geophys. Res.: Space Phys., 113(A5), A05307. https://doi.org/10.1029/2007JA012809

Tsunoda, R. T., Livingston, R. C., McClure, J. P., and Hanson, W. B. (1982). Equatorial plasma bubbles: vertically elongated wedges from the bottomside F layer. J. Geophys. Res.: Space Phys., 87(A11), 9171-9180. https://doi.org/10.1029/ja087ia11p09171

Tsunoda, R. T. (1985). Control of the seasonal and longitudinal occurrence of equatorial scintillations by the longitudinal gradient in integrated $E$ region pedersen conductivity. J. Geophys. Res.: Space Phys., 90(A1), 447-456. https://doi.org/10.1029/JA090iA01p00447

Vichare, G., and Richmond, A. D. (2005). Simulation study of the longitudinal variation of evening vertical ionospheric drifts at the magnetic equator during equinox. J. Geophys. Res.: Space Phys., 110(A5), A05304. https://doi.org/10.1029/2004JA010720

Woodman, R. F., \& Hoz, C. L. (1976). Radar observations of $f$ region equatorial irregularities. J. Geophys. Res.: Space Phys., 81(31). https://doi.org/10.1029/JA081i031p05447

Wu, K., Xu, J. Y., Wang, W. B., Sun, L. C., Liu, X., and Yuan, W. (2017). Interesting equatorial plasma bubbles observed by all-sky imagers in the equatorial region of China. J. Geophys. Res.: Space Phys., 122(10), 10596-10611. 
https://doi.org/10.1002/2017ja024561

Wu, K., Xu, J. Y., Xiong, C., and Yuan, W. (2018). Edge plasma enhancements of equatorial plasma depletions observed by all-sky imager and the C/NOFS satellite. J. Geophys. Res.: Space Phys., 123(10), 8835-8849.

https://doi.org/10.1029/2018ja025809

Wu, K., Xu, J. Y., Yue, X. N., Xiong, C., Wang, W. B., Yuan, W., Wang, C., Zhu, Y. J., and Ji, L. (2020). Equatorial plasma bubbles developing around sunrise observed by an all-sky imager and global navigation satellite system network during storm time. Ann. Geophys., 38(1), 163-177.

https://doi.org/10.5194/angeo-38-163-2020

Wu, K., Xu, J. Y., Wang, W. B., Sun, L. C., and Yuan, W. (2021). Interaction of oppositely traveling medium-scale traveling ionospheric disturbances observed in low latitudes during geomagnetically quiet nighttime. J. Geophys. Res.: Space Phys., 126(2), e2020JA028723.

https://doi.org/10.1029/2020JA028723
Xiong, C., Park, J., Lühr, H., Stolle, C., and Ma, S. Y. (2010). Comparing plasma bubble occurrence rates at CHAMP and GRACE altitudes during high and low solar activity. Ann. Geophys., 28(9), 1647-1658. https://doi.org/10.5194/angeo-28-1647-2010

Xiong, C., Lühr, H., and Fejer, B. G. (2015). Global features of the disturbance winds during storm time deduced from CHAMP observations. J. Geophys. Res.: Space Phys., 120(6), 5137-5150. https://doi.org/10.1002/2015JA021302

Yokoyama, T., Shinagawa, H., and Jin, H. (2014). Nonlinear growth, bifurcation, and pinching of equatorial plasma bubble simulated by three-dimensional high-resolution bubble model. J. Geophys. Res.: Space Phys., 119(12), 10474-10482. https://doi.org/10.1002/2014JA020708

Zalesak, S. T., Ossakow, S. L., and Chaturvedi, P. K. (1982). Nonlinear equatorial spread $F$ : the effect of neutral winds and background Pedersen conductivity. J. Geophys. Res.: Space Phys., 87(A1), 151-166. https://doi.org/10.1029/JA087iA01p00151 